Supporting Information: Kinetics of the one-pot transformation of citronellal to menthols on Ru/H-BEA catalysts

Jutta Plößer ${ }^{\mathrm{a}}$, Martin Lucasa ${ }^{\mathrm{a}}$, Johan Wärnå ${ }^{\mathrm{b}}$, Tapio Salmi ${ }^{\mathrm{b}}$, Dmitry Yu. Murzin ${ }^{\mathrm{b}}$ and Peter Claus $^{\mathrm{a}}$

${ }^{\text {a}}$ Technical University Darmstadt, Dept. Chemistry, Chemical Technology II, Alarich-WeissStraße 8, 64287 Darmstadt, Germany

${ }^{\mathrm{b}}$ Laboratory of Industrial Chemistry, Åbo Akademi University, Biskopsgatan 8, 20500 Åbo/Turku, Finland

*: Corresponding author: claus@tc2.tu-darmstadt.de

Tel.: +4961511623290

Fax: +496151 1623288 


\section{Diffusion Limitation}

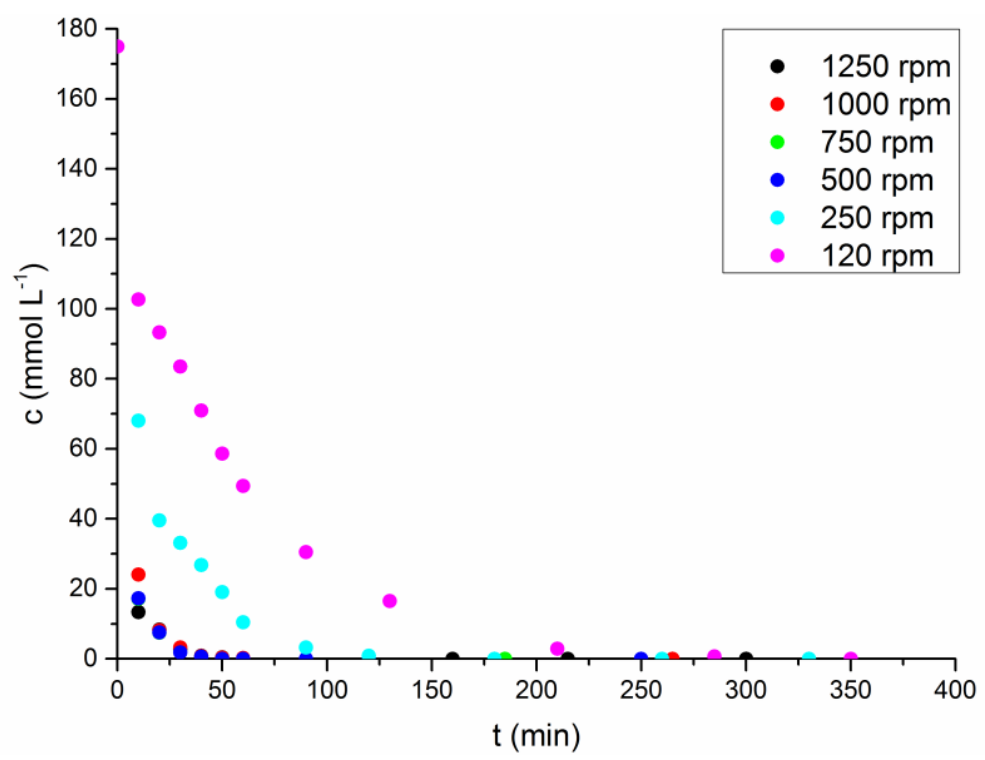

Figure S1: Influence of stirring rate on the conversion of citronellal on $1 \% \mathrm{Ru} / \mathrm{H}-\mathrm{BEA}-25$.

Reaction conditions: $\mathrm{c}_{\mathrm{CAL}}=0,175 \mathrm{~mol} \mathrm{~L}{ }^{-1} ; 150 \mathrm{~mL}$ h-hexane; $1 \mathrm{~mL}$-tetradecane as internal GC standard; 0,5 g 1\%Ru/H-BEA-25; 25 bar; $373 \mathrm{~K}$.

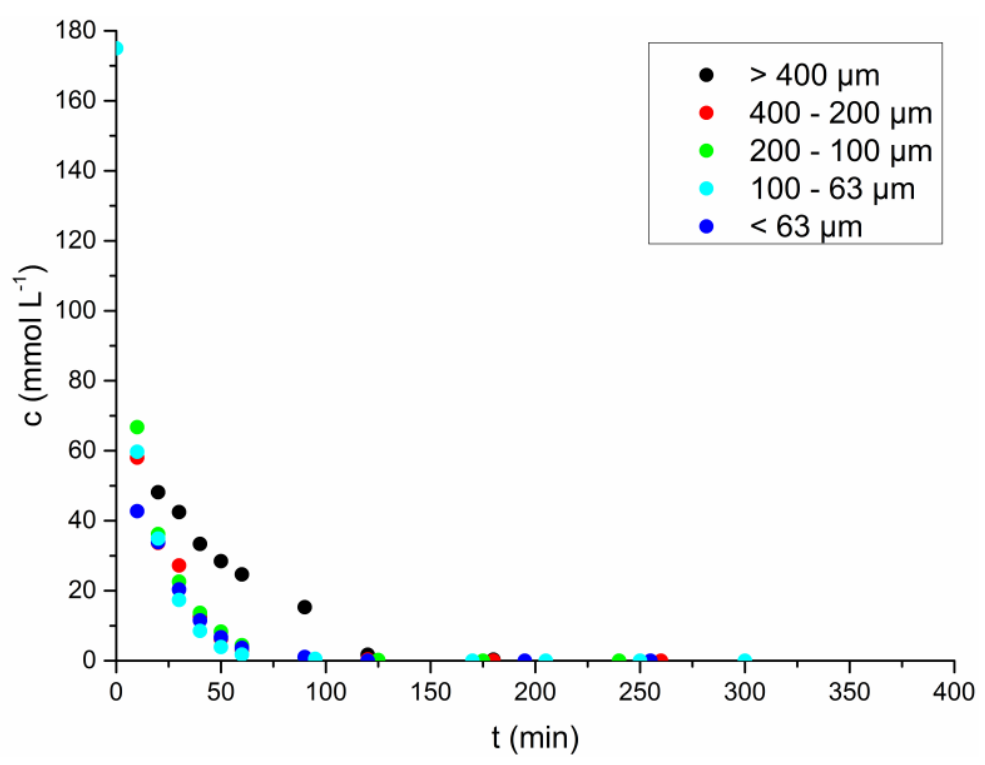

Figure S2: Influence of catalyst particle size on the conversion of citronellal on $1 \% \mathrm{Ru} / \mathrm{H}-$ BEA-25. Reaction conditions: $\mathrm{c}_{\mathrm{CAL}}=0,175 \mathrm{~mol} \mathrm{~L}^{-1} ; 150 \mathrm{~mL}$ h-hexane; $1 \mathrm{~mL}$-tetradecane as internal GC standard; 0,5 g 1\%Ru/H-BEA-25; 25 bar; $373 \mathrm{~K}$. 


\section{MCMC Sensitivity plots}

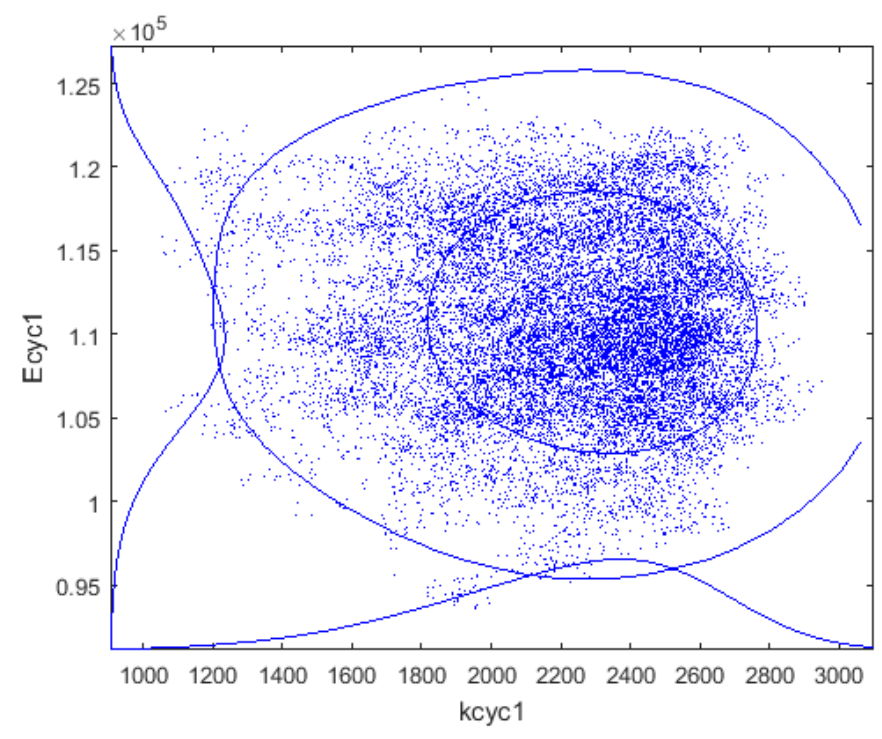

Figure S3: MCMC sensitivity plot Ecyc1 vs. kcyc1.

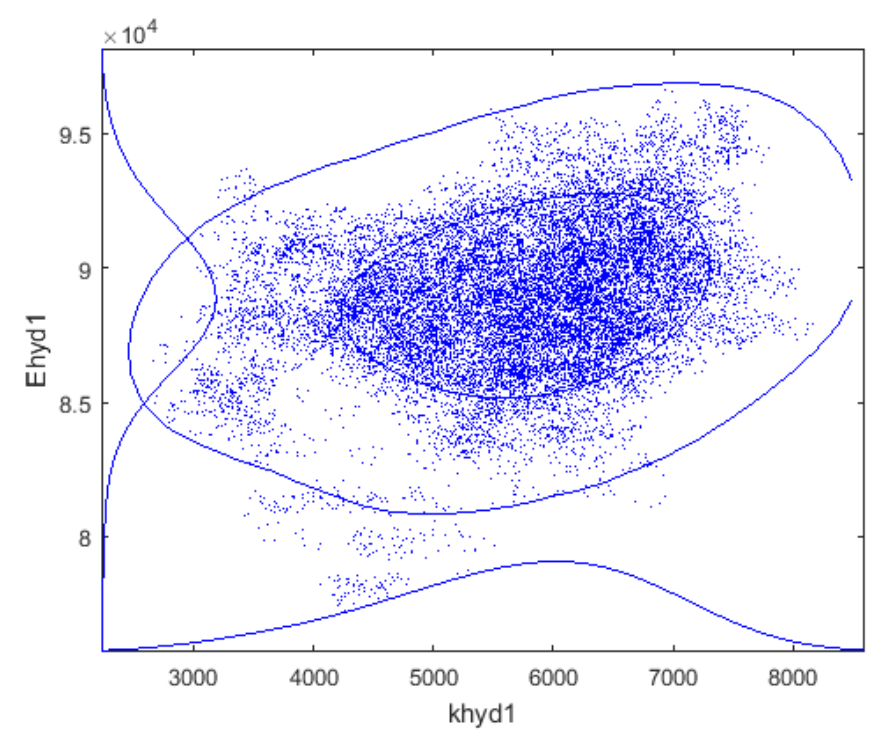

Figure S4: MCMC sensitivity plot Ehyd1 vs. khyd1. 


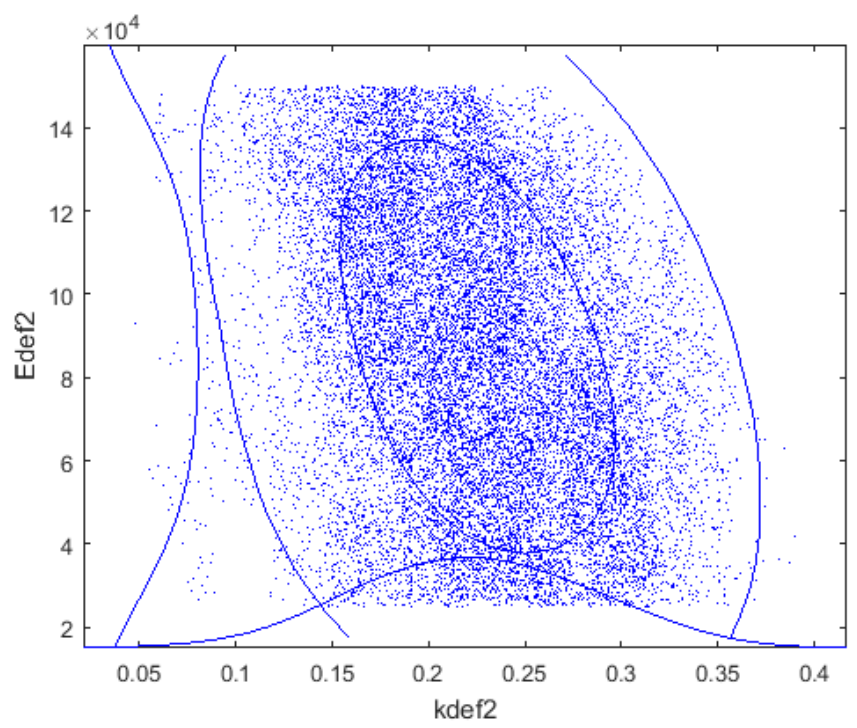

Figure S5: MCMC sensitivity plot Edef2 vs. kdef2.

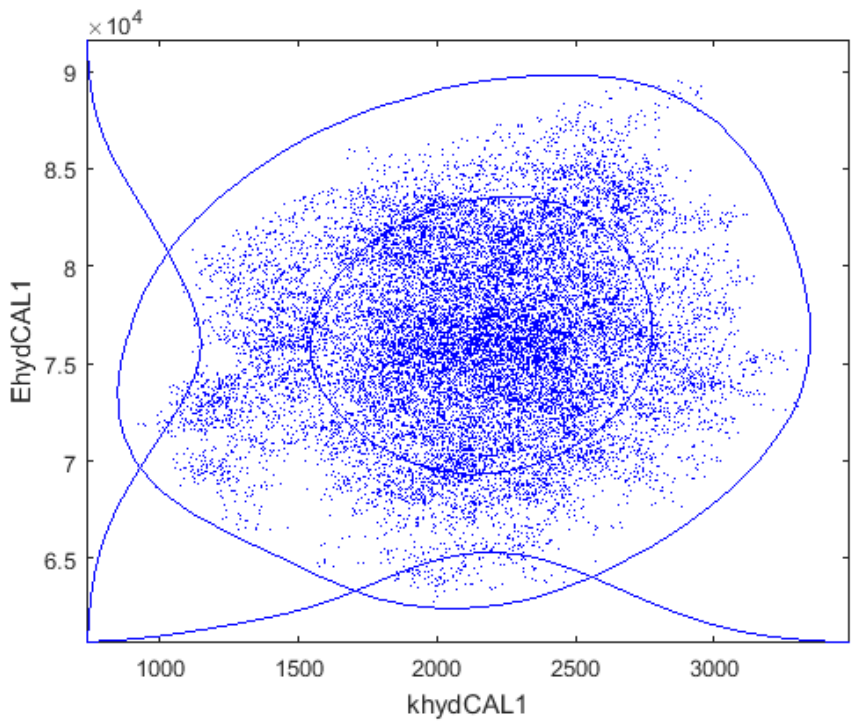

Figure S6: MCMC sensitivity plot EhydCAL1 vs. khydCAL1. 


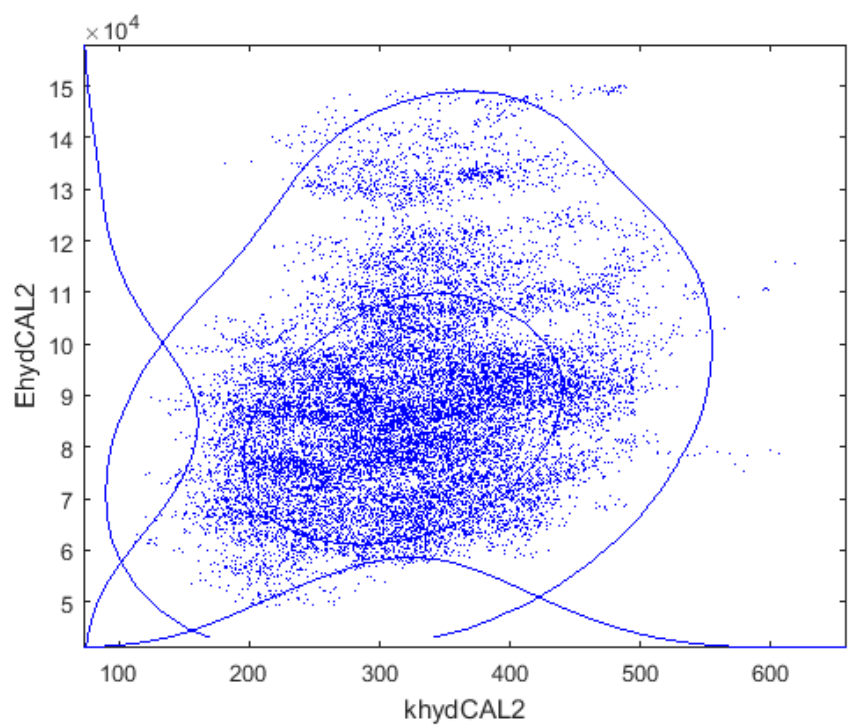

Figure S7: MCMC sensitivity plot EcycCAL2 vs. kcycCAL2.

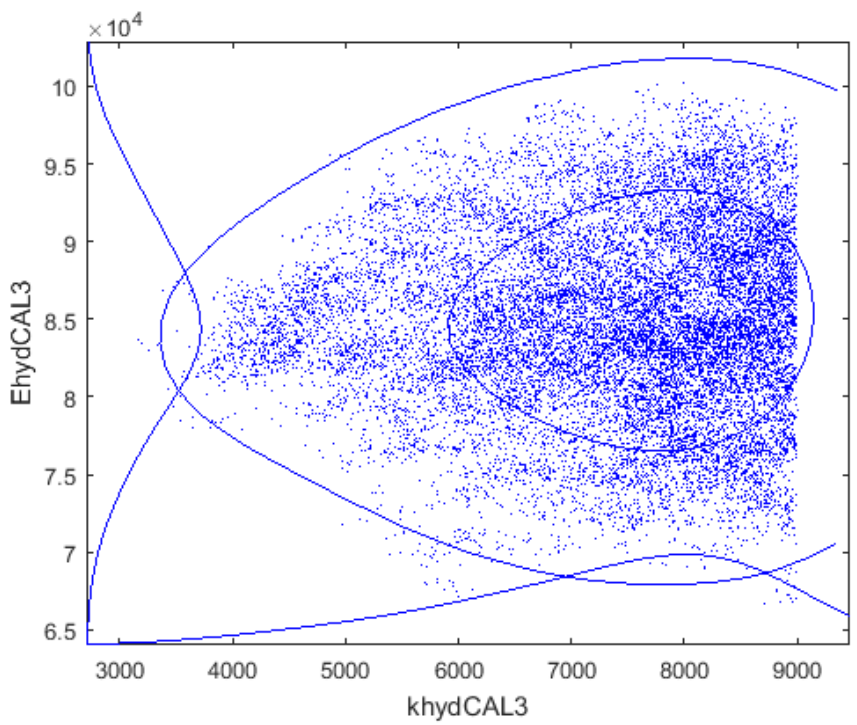

Figure S8: MCMC sensitivity plot EcycCAL2 vs. khydCAL2. 


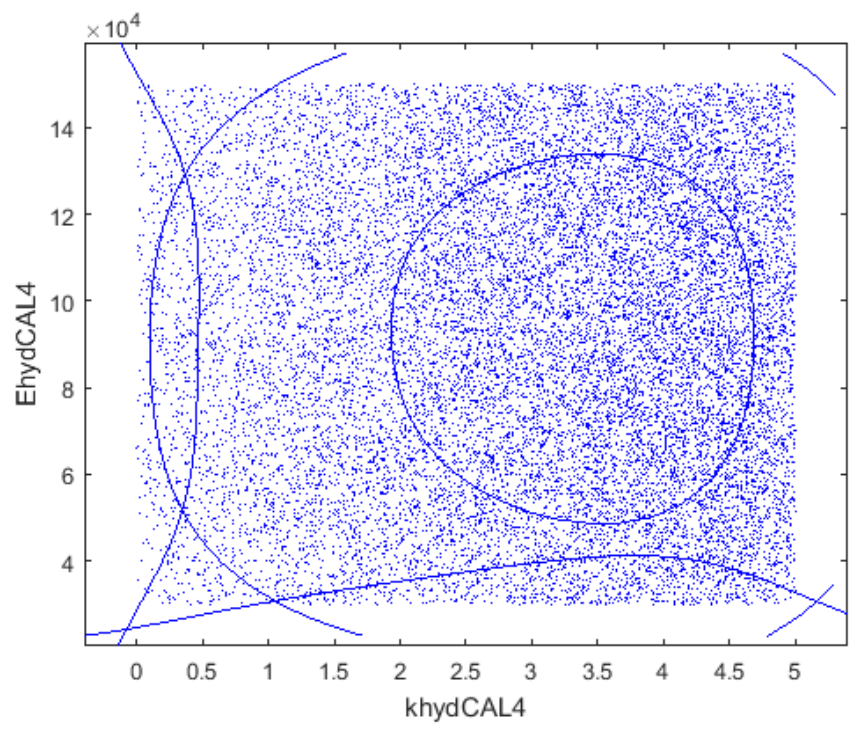

Figure S9: MCMC sensitivity plot KCAL2 vs. kcyc1.

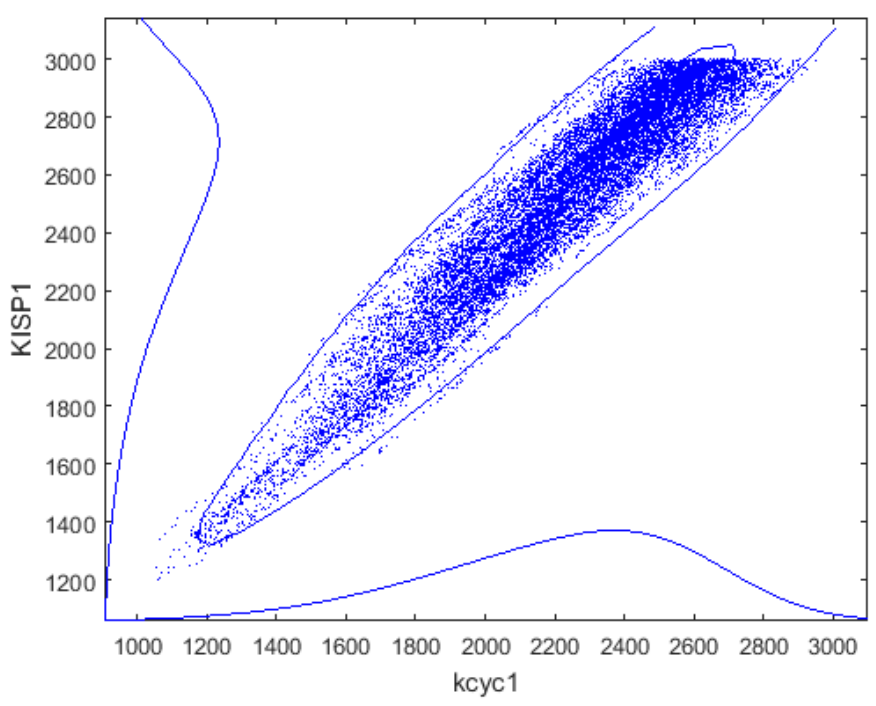

Figure S10: MCMC sensitivity plot KISP1 vs. kcyc1. 


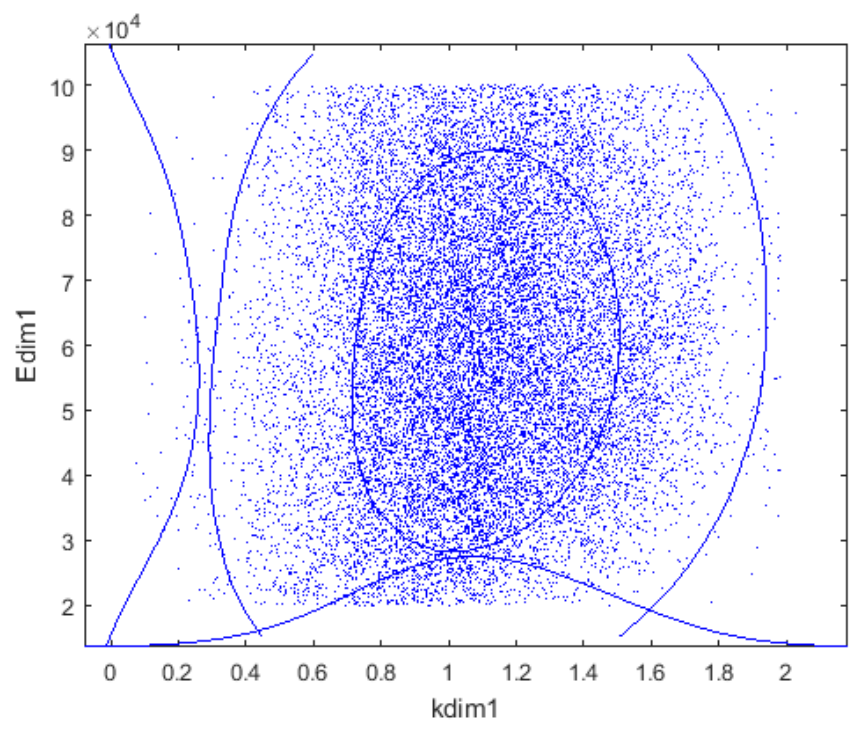

Figure S11: MCMC sensitivity plot Edim1 vs. kdim1.

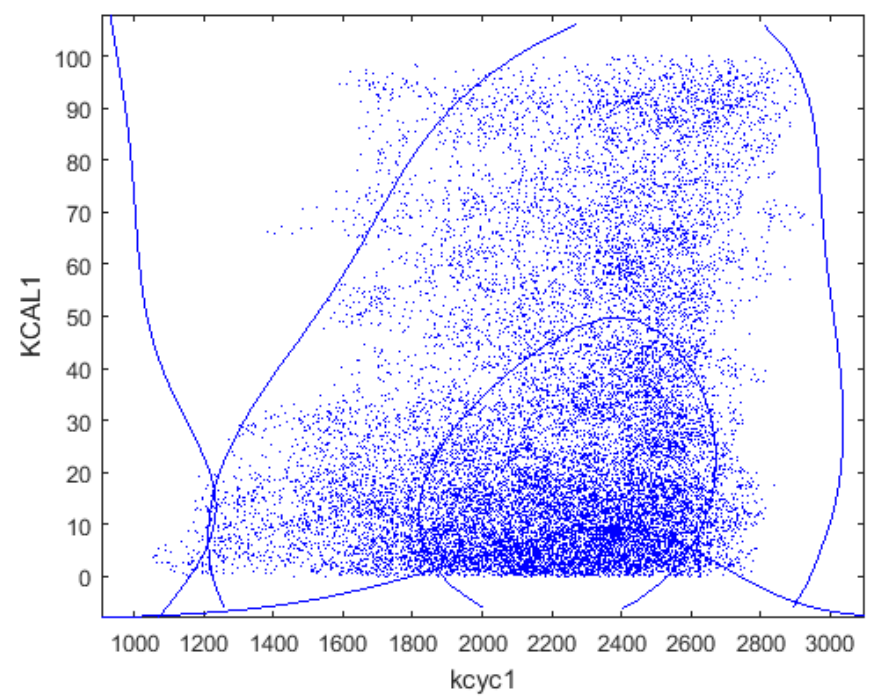

Figure S12: MCMC sensitivity plot KCAL1 vs. kcyc1. 


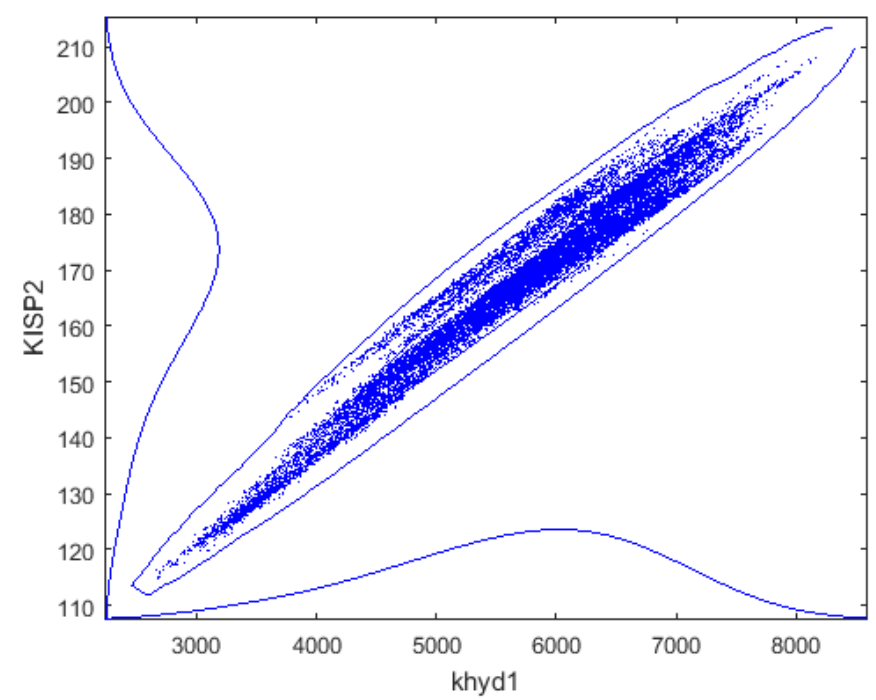

Figure S13: MCMC sensitivity plot KISP2 vs. khyd1.

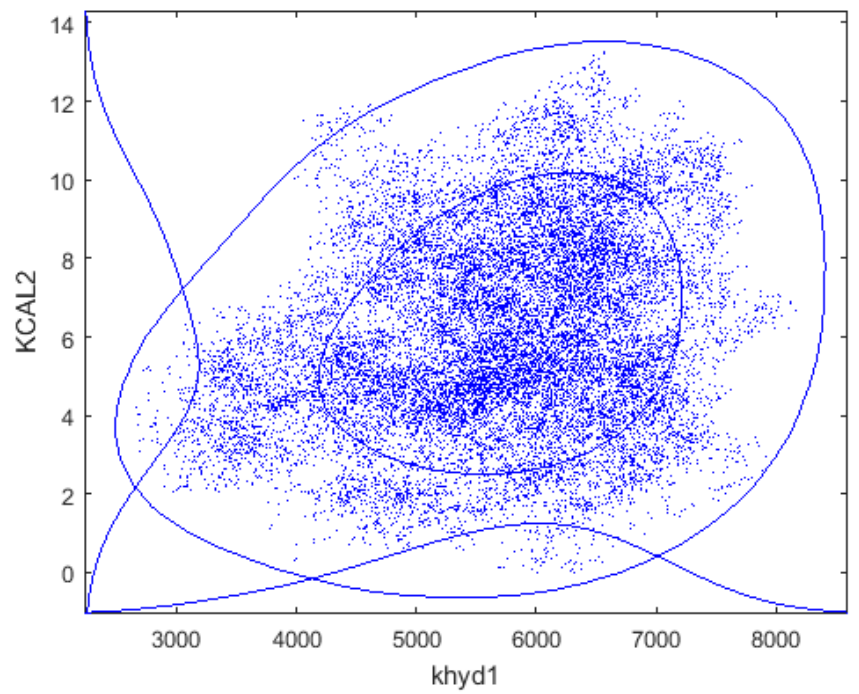

Figure S14: MCMC sensitivity plot KCAL2 vs. khyd1. 


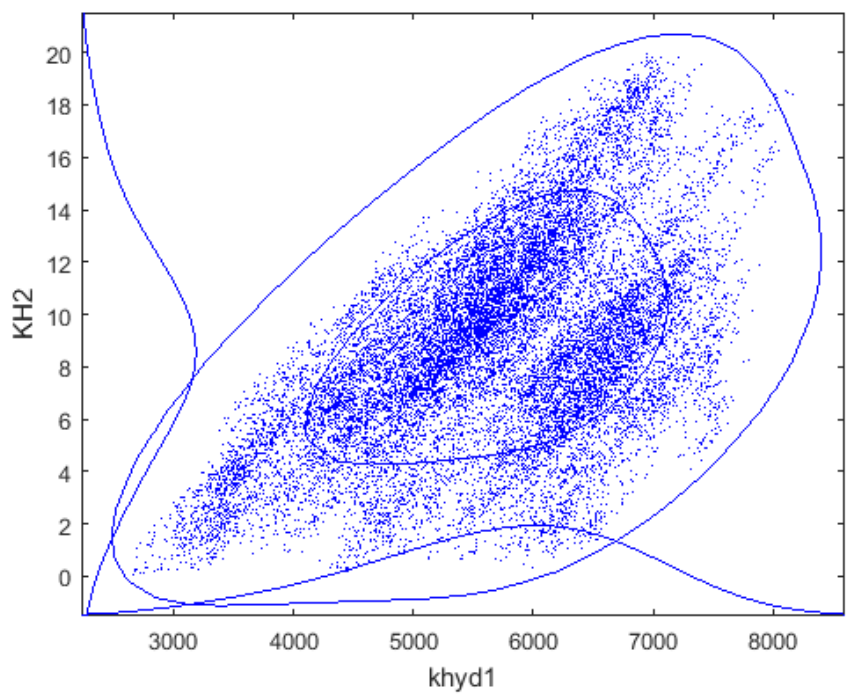

Figure S15: MCMC sensitivity plot KH2 vs. khyd1.

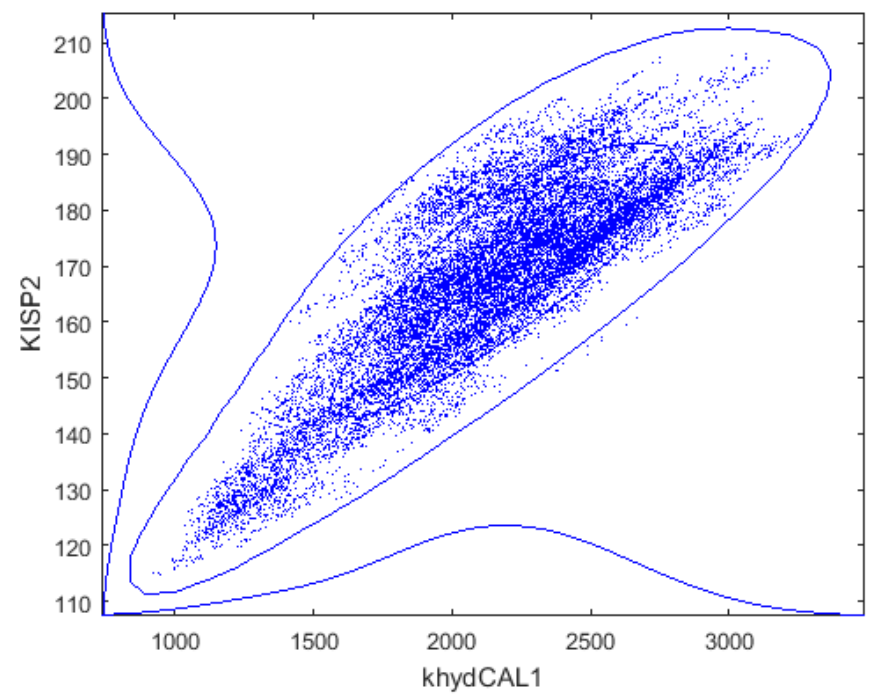

Figure S16: MCMC sensitivity plot KISP2 vs. khydCAL1. 


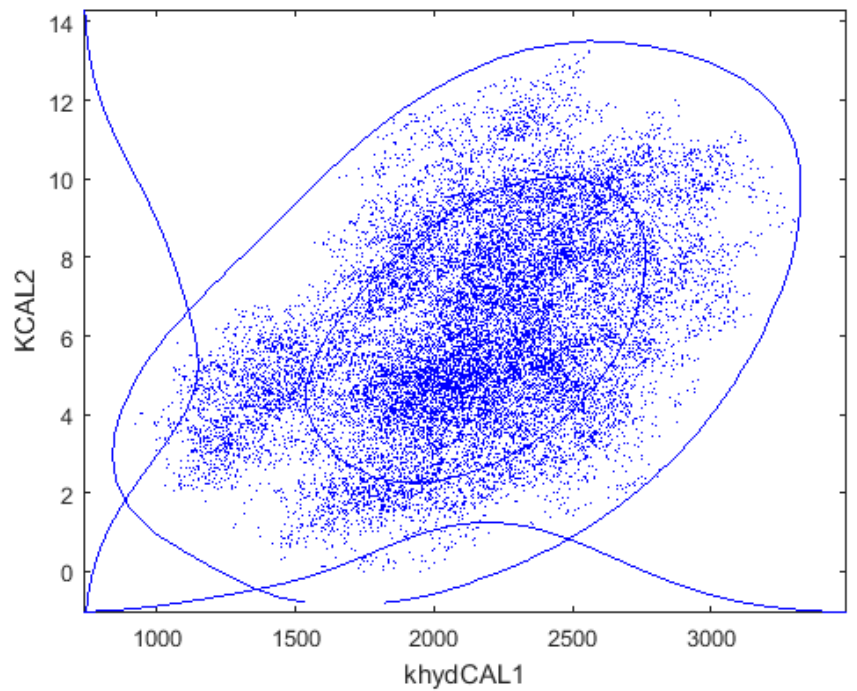

Figure S17: MCMC sensitivity plot KCAL2 vs. khydCAL1.

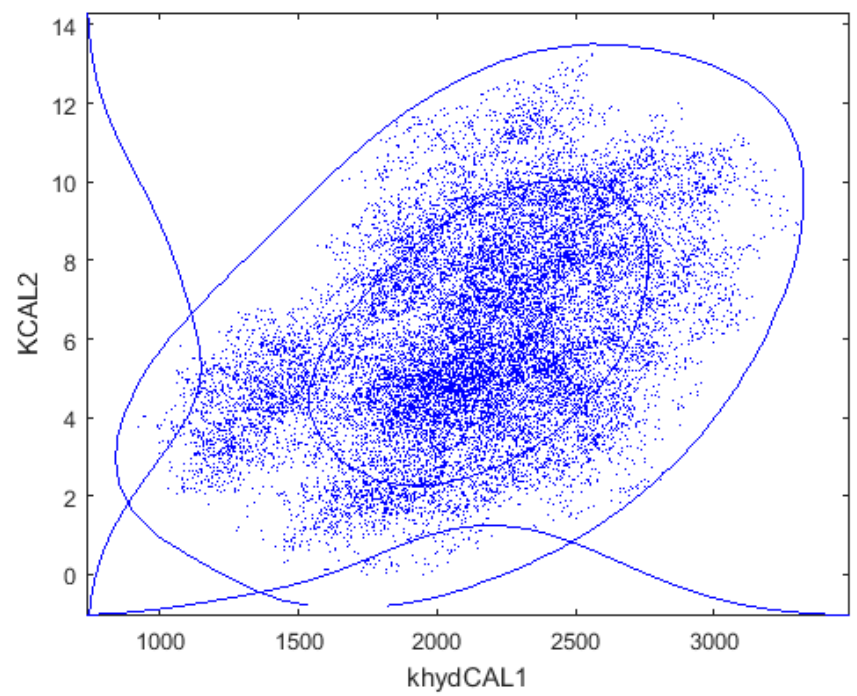

Figure S18: MCMC sensitivity plot KH2 vs. khydCAL1. 


\section{Correlation matrix}

\begin{tabular}{|c|c|c|c|c|c|c|c|c|c|c|c|c|c|c|c|c|c|c|c|c|c|}
\hline & kcyc1 & & & & & & & & & & & & & & & & & & & & \\
\hline kcyc1 & 1,00 & khyd1 & & & & & & & & & & & & & & & & & & & \\
\hline khyd1 & 0,30 & 1,00 & kdef2 & & & & & & & & & & & & & & & & & & \\
\hline kdef2 & 0,25 & 0,28 & 1,00 & khyd & CAL1 & & & & & & & & & & & & & & & & \\
\hline khydCAL1 & 0,37 & 0,97 & 0,27 & 1,00 & khyd & CAL2 & & & & & & & & & & & & & & & \\
\hline khydCAL2 & 0,32 & 0,91 & 0,23 & 0,92 & 1,00 & khyd & CAL3 & & & & & & & & & & & & & & \\
\hline khydCAL3 & 0,33 & 0,96 & 0,26 & 0,95 & 0,89 & 1,00 & khyd & CAL4 & & & & & & & & & & & & & \\
\hline khydCAL4 & 0,29 & 0,32 & $-0,06$ & 0,35 & 0,47 & 0,32 & 1,00 & kdim1 & & & & & & & & & & & & & \\
\hline kdim1 & 0,08 & 0,17 & 0,03 & 0,17 & 0,21 & 0,16 & $-0,02$ & 1,00 & KCAL1 & & & & & & & & & & & & \\
\hline KCAL1 & 0,31 & 0,65 & 0,17 & 0,63 & 0,62 & 0,63 & 0,78 & $-0,20$ & 1,00 & KCAL2 & & & & & & & & & & & \\
\hline KCAL2 & $-0,01$ & 0,52 & 0,05 & 0,59 & 0,58 & 0,54 & 0,45 & $-0,08$ & 0,62 & 1,00 & KISP1 & & & & & & & & & & \\
\hline KISP1 & 0,99 & 0,26 & 0,24 & 0,33 & 0,28 & 0,29 & 0,24 & 0,10 & 0,24 & $-0,02$ & 1,00 & KISP2 & & & & & & & & & \\
\hline KISP2 & 0,29 & 1,00 & 0,28 & 0,97 & 0,89 & 0,95 & 0,25 & 0,19 & 0,60 & 0,48 & 0,25 & 1,00 & $\mathrm{KH} 2$ & & & & & & & & \\
\hline $\mathrm{KH} 2$ & 0,45 & 0,92 & 0,27 & 0,91 & 0,90 & 0,89 & 0,59 & 0,11 & 0,76 & 0,43 & 0,39 & 0,90 & 1,00 & Ecyc1 & & & & & & & \\
\hline Ecyc1 & 0,19 & $-0,09$ & 0,03 & $-0,06$ & $-0,07$ & $-0,07$ & $-0,13$ & 0,08 & $-0,20$ & $-0,26$ & 0,19 & $-0,07$ & $-0,06$ & 1,00 & Ehyd1 & & & & & & \\
\hline Ehyd1 & 0,12 & 0,01 & 0,04 & 0,00 & $-0,03$ & 0,01 & $-0,17$ & 0,10 & $-0,15$ & $-0,23$ & 0,13 & 0,03 & 0,00 & 0,59 & 1,00 & Edef2 & & & & & \\
\hline Edef2 & $-0,07$ & $-0,02$ & $-0,45$ & $-0,02$ & $-0,01$ & $-0,02$ & 0,09 & $-0,01$ & 0,02 & 0,06 & $-0,07$ & $-0,03$ & $-0,02$ & $-0,02$ & 0,00 & 1,00 & Ehydcal1 & & & & \\
\hline Ehydcal1 & 0,04 & 0,05 & 0,05 & 0,06 & 0,00 & 0,04 & $-0,18$ & 0,09 & $-0,15$ & $-0,32$ & 0,03 & 0,07 & 0,05 & 0,79 & 0,35 & $-0,04$ & 1,00 & Ehydcal2 & & & \\
\hline Ehydcal2 & $-0,02$ & 0,00 & 0,00 & $-0,01$ & 0,14 & 0,00 & 0,05 & 0,11 & $-0,06$ & $-0,14$ & $-0,03$ & 0,01 & 0,06 & 0,41 & 0,09 & $-0,02$ & 0,36 & 1,00 & Ehydcal3 & & \\
\hline Ehydcal3 & 0,02 & 0,08 & 0,04 & 0,05 & 0,02 & 0,19 & $-0,12$ & 0,06 & $-0,05$ & $-0,20$ & 0,01 & 0,09 & 0,07 & 0,41 & 0,21 & $-0,01$ & 0,43 & 0,14 & 1,00 & Ehydcal4 & \\
\hline Ehydcal4 & $-0,07$ & $-0,07$ & 0,03 & $-0,09$ & $-0,08$ & $-0,07$ & $-0,27$ & 0,02 & $-0,22$ & $-0,12$ & $-0,06$ & $-0,05$ & $-0,14$ & 0,09 & 0,08 & $-0,03$ & 0,02 & 0,13 & 0,03 & 1,00 & Edim1 \\
\hline Edim1 & $-0,01$ & 0,05 & 0,00 & 0,04 & 0,04 & 0,04 & 0,01 & 0,03 & 0,02 & 0,01 & $-0,02$ & 0,05 & 0,04 & $-0,06$ & 0,20 & 0,04 & $-0,03$ & 0,02 & 0,02 & $-0,02$ & 1,00 \\
\hline
\end{tabular}

